\title{
Fiber Optic 3-Component Seismometer
}

\author{
Jing HAN, Wentao ZHANG ${ }^{*}$, Dongshan JIANG, Zhaogang WANG, and Fang LI \\ Optoelectronic System Laboratory, Institute of Semiconductors, Chinese Academy of Sciences, No. 35, Qinghua East \\ Road, Hai dian District Beijing, 100083, China \\ *Corresponding author: Wentao ZHANGＥ-mail: zhangwt@semi.ac.cn
}

\begin{abstract}
An all-metal 3-component optical fiber seismometer was proposed and experimentally demonstrated. The theoretical analysis was given based on the electro-mechanical theory. Calibration results showed that the axis sensitivity was about $41 \mathrm{~dB}$ (re: $0 \mathrm{~dB}=1 \mathrm{rad} / \mathrm{g}$ ) with a fluctuation $\pm 2 \mathrm{~dB}$ in the frequency bandwidth of $5 \mathrm{~Hz}-400 \mathrm{~Hz}$. A transverse sensitivity of about $-40 \mathrm{~dB}$ was achieved. The fluctuation of the acceleration sensitivity for the three accelerometers in the seismometer was within $\pm 2.5 \mathrm{~dB}$. The minimum phase demodulation detection accuracy of the phase-generated carrier (PGC) was $10^{-5} \mathrm{rad} / \sqrt{ } \mathrm{Hz}$, and the minimum detectable acceleration was calculated to be $90 \mathrm{ng} / \sqrt{\mathrm{Hz}}$.
\end{abstract}

Keywords: Fiber optic, seismometer, transverse sensitivity, accelerometer

Citation: Jing HAN, Wentao ZHANG, Dongshan JIANG, Zhaogang WANG, and Fang LI, "Fiber Optic 3-Component Seismometer," Photonic Sensors, 2014, 4(2): 102-107.

\section{Introduction}

Compared to the conventional electrical accelerometer, the optical fiber accelerometer is widely used in oil or gas exploration[1], earth quake monitoring [2] due to its high sensitivity, wide dynamic range, and immunity to the electro magnetic interference (EMI). And the optical fiber accelerometer is also widely used in target discrimination, oil well logging [3], and permanent reservoir monitoring[4]. The multi-component detection and the low transverse sensitivity are necessary for large scale array applications. Currently, the design of the interferometric optical fiber accelerometer mainly includes two types: compliant mandrel and flexural disc. The compliant material is used as the sensing element in the compliant mandrel accelerometer. The long-time performance of the rubber mandrel needs to be further investigated [5]. The design of the disc type accelerometer exhibits relatively poor suppression of the transverse responsivity [6-10]. Moreover, the consistency of the acceleration sensitivity and reliability is important for the large-scale optical fiber seismometer array.

In this paper, the design and performances of a double metal diaphragm-based optical fiber 3-component seismometer with the low transverse sensitivity and fiber coils wrapped on an all-metal structure is proposed. The theoretical evaluation and the experimental results are both given.

\section{Principles of the seismometer}

The proposed 3-component seismometer consists of three optical fiber accelerometers. The optical fiber accelerometer is shown in Fig. 1. Two clamped metal diaphragms are used as the elastic element. The movable mass and movable bridge are inertial elements. The sensing arm of the optical fiber Michelson interferometer is wrapped around

Received:18 December 2013 / Revised version: 5 January 2014

(C) The Author(s) 2014. This article is published with open access at Springerlink.com

DOI: $10.1007 / \mathrm{s} 13320-014-0173-4$

Article type: Regular 
the surface of the upper moving bridge and the lower stationary bridge with a certain pre-strain. When the sensor is accelerated, the movable mass and movable bridge will have a displacement relative to the stationary bridge and make the metal diaphragms deformed. Thus, the sensing optical fiber will be compressed or extended, and the optical path length will change, leading to the phase shift in the optical fiber interferometer. The Michelson interferometric system is shown in Fig. 2. The phase shift can be demodulated by the phase generated carrier (PGC) technology [11], and finally the acceleration is extracted.

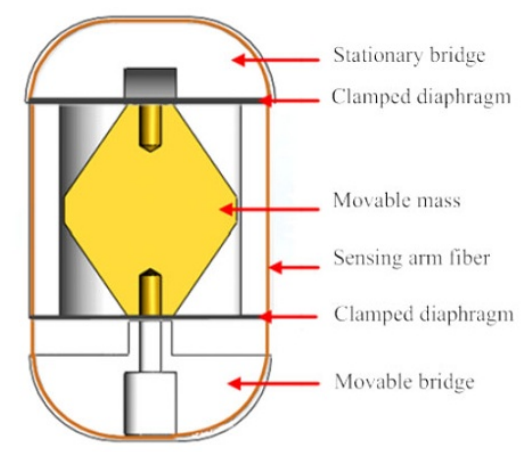

Fig. 1 Schematic diagram of the optical fiber accelerometer.

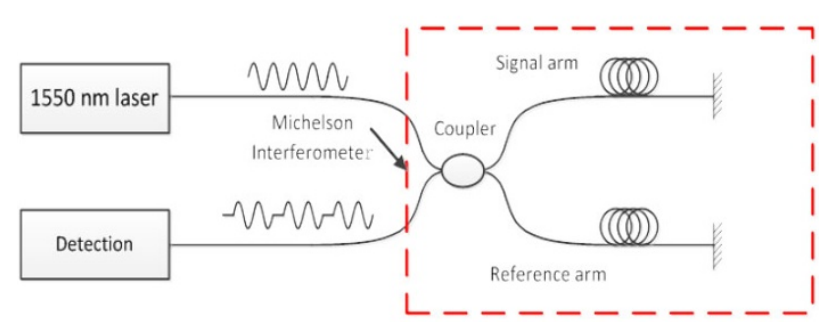

Fig. 2 Structure diagram of the Michelson interferometric system.

Two clamped metal diaphragms at the both ends of the mass restrict the lateral movement of the mass, which reduces the transverse sensitivity of the designed accelerometer. The designed accelerometer is expected to improve the reliability of long-term using in harsh environments due to its all-mental design.

Based on the electro-mechanical theory, the sensitivity and the frequency can be analyzed. Figure 3 shows the electric-circuit element analogous to the proposed accelerometer. As shown in Fig. 3(a), the vibration system is constituted of the spring (metal diaphragm and optical fibers) and the mass moved by the acceleration signal. The line of force starts from the contact between the spring and the housing and reaches the mass through the spring, where the line of force is divided into two. One balance in inertial force is through the mass and ends in the rigid housing. The other reaches internal air through the mechanical resistance. In the admittance type of the electric-mechanical analog model, the equivalent mechanical compliance $C_{m}$ and $C_{m 2}$ of the metal diaphragm and optical fibers can be expressed as the inductance of the circuit, the equivalent mechanical mass $M_{m}, M_{m 1}$, and $M_{m 2}$ of the mass, metal diaphragm, and optical fibers can be expressed as the capacitance of the circuit, and the mechanical conductance $G_{m}$ can be expressed as the resistance of the circuit. The mechanical elements in the path of the force line are replaced by the circuit analog symbol and can obtain the equivalent circuit, as shown in Fig. 3(b).

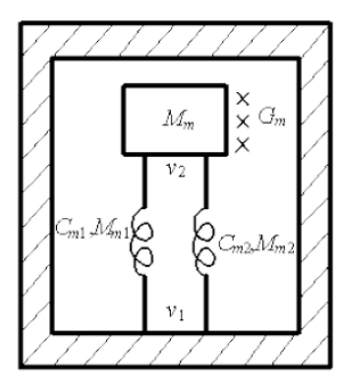

(a)

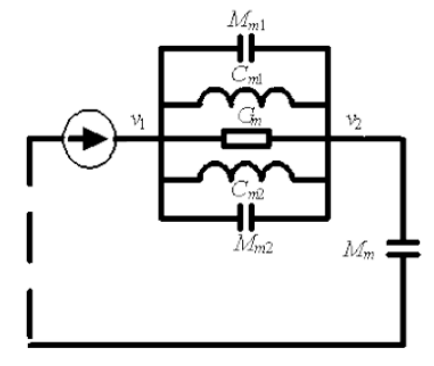

(b)
Fig. 3 Schematic of the proposed accelerometer: (a) geometrical configuration and (b) equivalent circuit.

Under the function of acceleration, the equivalent circuit equations of mechanical vibration system can be written as

$$
\left(v_{1}-v_{2}\right)\left(\frac{1}{j \omega C_{m 1}}+\frac{1}{G_{m}}+\frac{1}{j \omega C_{m 2}}\right)=j \omega M_{m} v_{2}
$$

where $M_{m}, M_{m 1}$, and $M_{m 2}$ are the equivalent mechanical mass of the mass, metal diaphragm, and optical fibers, respectively; $C_{m 1}$ and $C_{m 2}$ are the equivalent mechanical compliance of the metal 
diaphragm and optical fibers, respectively; $G_{m}$ is the mechanical conductance of the vibration system; $\omega$ is the angular frequency of acceleration.

In the system based on (1), $a=a_{0} e^{j \omega t}$ is the acceleration, and $a_{0}$ is the amplitude of the acceleration. The relative displacement between the mass and the base $\varepsilon$ can be expressed as

$$
\varepsilon=-\frac{j M_{m} a}{Z_{m} \omega}=\varepsilon_{a} e^{j\left(\omega t-\theta_{0}-\frac{\pi}{2}\right)}
$$

where

$$
\begin{gathered}
\varepsilon_{a}=\frac{M_{m} a_{0}}{\omega\left|Z_{m}\right|} \\
\left|Z_{m}\right|=\sqrt{\left(\frac{1}{G_{m}}\right)^{2}+\left(\omega M_{m}-\frac{C_{m 1}+C_{m 2}}{\omega C_{m 1} C_{m 2}}\right)^{2}} .
\end{gathered}
$$

The equivalent mechanical compliance of the metal diaphragm and optical fibers $C_{m 1}, C_{m 2}$ can readily be shown to be

$$
\begin{gathered}
C_{m 1}=\frac{L^{3}+r^{3}-2 r^{2} L}{8 E b h^{3}} \\
C_{m 2}=\frac{1}{4 N E_{f} A}
\end{gathered}
$$

where $E, L, b$, and $h$ are the Young's modulus, length, width, and height of the rectangular diaphragm, respectively; $E_{f}$ and $A$ are the Young's modulus and sectional area of the optical fibers; $r$ is the doublication radius between the rectangle diaphragm and the mass; $N$ is the number of turns of the optical fibers; $l$ is the length of a circle of the optical fiber.

The change in the fiber optic signal arm length leads to the phase delay $\Delta \varphi$, which can be written as

$$
\Delta \varphi=2\left(1-p_{e}\right) n k N \varepsilon_{a}
$$

where $p_{\mathrm{e}}=0.22$ and $n$ are the elastic-optic coefficient and refractive index of optical fibers; $k$ is the wave number of the optical signal.

In conclusion, the acceleration sensitivity of the optical fiber accelerometer can be expressed as

$$
M_{a}=\frac{\Delta \varphi}{a}=\frac{2 \times 0.87 n k N M_{m}}{\omega \sqrt{\left(\frac{1}{G_{m}}\right)^{2}+\left(\omega M_{m}-\frac{C_{m 1}+C_{m 2}}{\omega C_{m 1} C_{m 2}}\right)^{2}}} .
$$

When the equivalent mechanical resistance of the vibration system can be ignored, $G_{m}$ tends to $\infty$, and then the equation can be simplified as

$$
s_{a}=\frac{s_{0}}{1-\frac{f^{2}}{f_{0}^{2}}}
$$

where $s_{0}$ and $f_{0}$ are the acceleration sensitivity and resonance frequency of optical fiber accelerometer, respectively.

$$
\begin{gathered}
s_{0}=\frac{2 \times 0.87 n k N M_{m}}{\frac{C_{m 1}+C_{m 2}}{C_{m 1} C_{m 2}}} \\
f_{0}=\frac{1}{2 \pi} \times \sqrt{\frac{C_{m 1}+C_{m 2}}{m C_{m 1} C_{m 2}}} .
\end{gathered}
$$

It can be found from (9) that the acceleration sensitivity and resonance frequency can be simultaneously increased by increasing the number of turns of the optical fiber in the case of not increasing the accelerometer size at the mass of a certain value. The encapsulation of the accelerometer is shown in Fig. 4. The axial sensitivity of the proposed accelerometer was calculated to be about $40 \mathrm{~dB}$ (re: $0 \mathrm{~dB}=1 \mathrm{rad} / \mathrm{g}$ ).

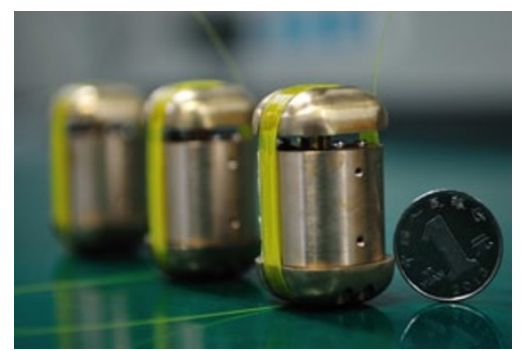

Fig. 4 Encapsulation of accelerometer.

\section{Experiment and results}

The proposed optical fiber accelerometers were tested to evaluate their performances. The measurement system is shown in Fig. 5. A precision shaking table (type BKV650) provided a series of sine excitations with the tunable frequency as the 
input signal. A standard piezoelectric accelerometer (type BK4371) was used as the reference. The output of the optical fiber accelerometer was demodulated by using the PGC method [12].

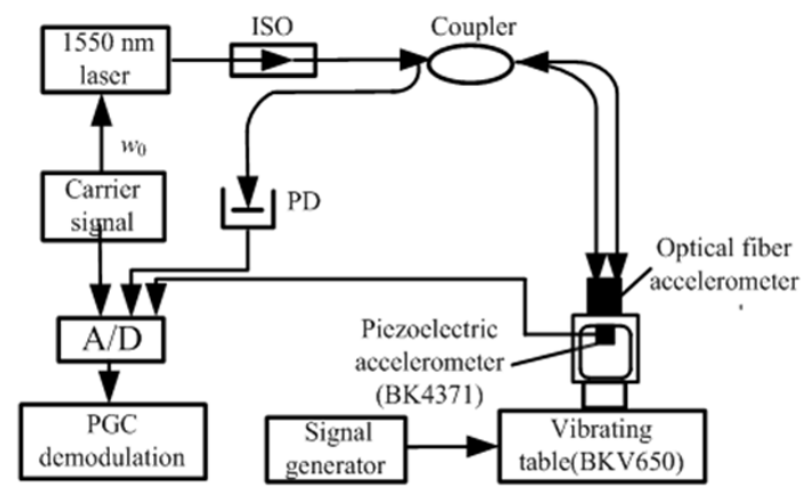

Fig. 5 Experimental setup of the optical fiber accelerometer.

The comparative method was adopted to conduct the optical fiber accelerometer performance test. The special clamp has been designed in order to make the standard piezoelectric accelerometer, and the optical fiber accelerometer had the same vibration signal on the precision shaking table.

\subsection{Time-domain demodulation of the accelerometer}

When the frequency of the vibration table signal was $150 \mathrm{~Hz}$, the time-domain as the result of the PGC demodulation is shown in Fig. 6. The result shows that the design of the optical fiber accelerometer can achieve accurate measurements of the acceleration signal demodulation of frequency and maintain high standards of the piezoelectric acceleration sensor in the time-domain synchronization.

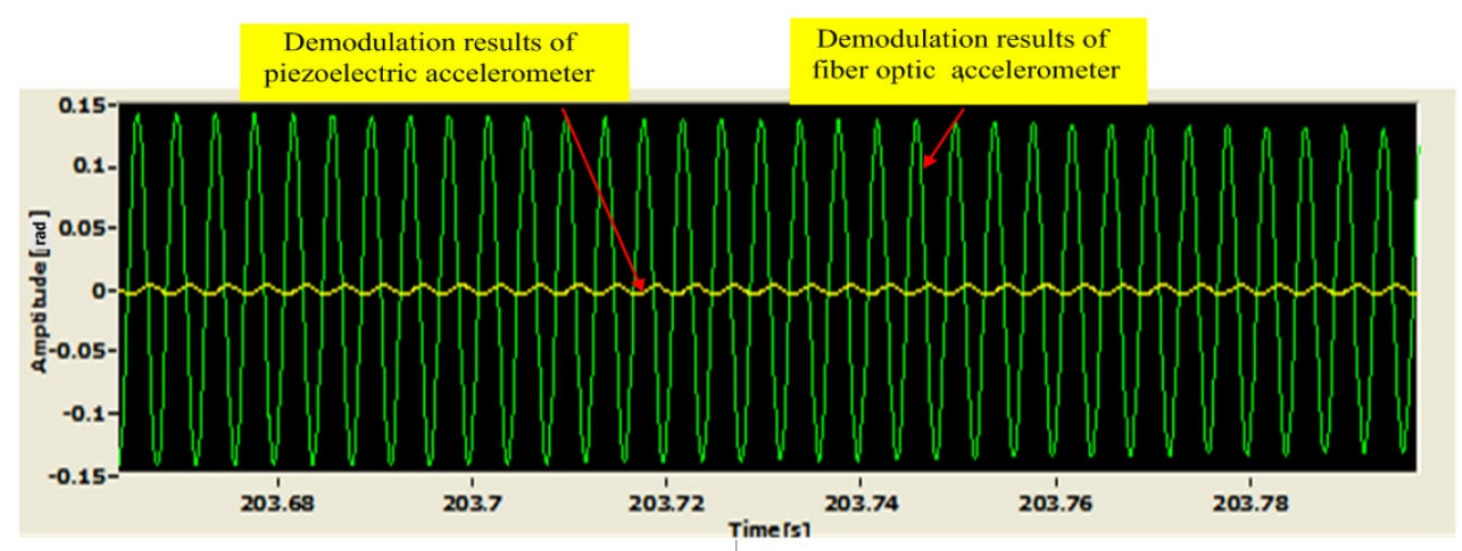

Fig. 6 Time-domain graph of the measured accelertion signal at $150 \mathrm{~Hz}$.

\subsection{Linearity of the acceleration response}

In the test, the acceleration signal frequency was $100 \mathrm{~Hz}$, and the sine signal amplitude of the signal generator was in the range of $0.01 \mathrm{~m} / \mathrm{s}^{2}-0.45 \mathrm{~m} / \mathrm{s}^{2}$. The output of the phase demodulation system and

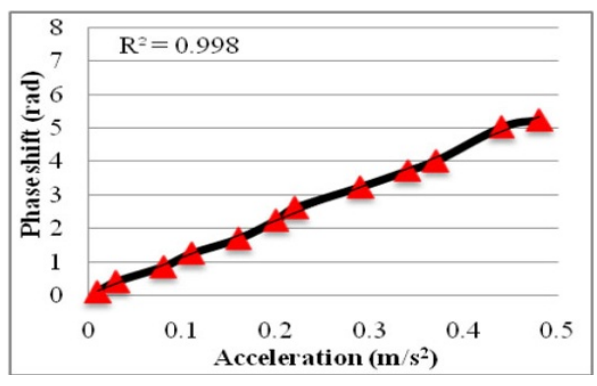

Fig. 7 Linearity acceleration sensor test. the test results of the acceleration amplitude are shown in Fig. 7. It can be seen from the linear fitting results that the design of the optical fiber accelerometer showed the good linear relationship, and the correlation coefficient was 0.998 .

\subsection{Axial and across acceleration responses}

Figures 8 and 9 show the frequency responses of the axial and transverse sensitivities. The axial sensitivity was about $41 \mathrm{~dB}$ (re: $0 \mathrm{~dB}=1 \mathrm{rad} / \mathrm{g}$ ), with a fluctuation $\pm 2 \mathrm{~dB}$ in the frequency bandwidth of $5 \mathrm{~Hz}-400 \mathrm{~Hz}$, and the resonance frequency was about $630 \mathrm{~Hz}$, which were in agreement with the result of the theoretical analysis. It can be also found 
that the transverse sensitivity was about $3 \mathrm{~dB}$ (re: $0 \mathrm{~dB}=1 \mathrm{rad} / \mathrm{g}$ ), with a fluctuation $\pm 1.5 \mathrm{~dB}$ in the frequency bandwidth of $5 \mathrm{~Hz}-400 \mathrm{~Hz}$, which means that a reduction in the transverse sensitivity of about $-40 \mathrm{~dB}$ was achieved.

The noise level of the test system was lower than $10^{-5} \mathrm{rad} / \mathrm{Hz}^{1 / 2}$ within $5 \mathrm{~Hz}-1 \mathrm{kHz}$, which resulted in a minimum detectable acceleration of $90 \mathrm{ng} / \mathrm{Hz}^{1 / 2}$ in theory.

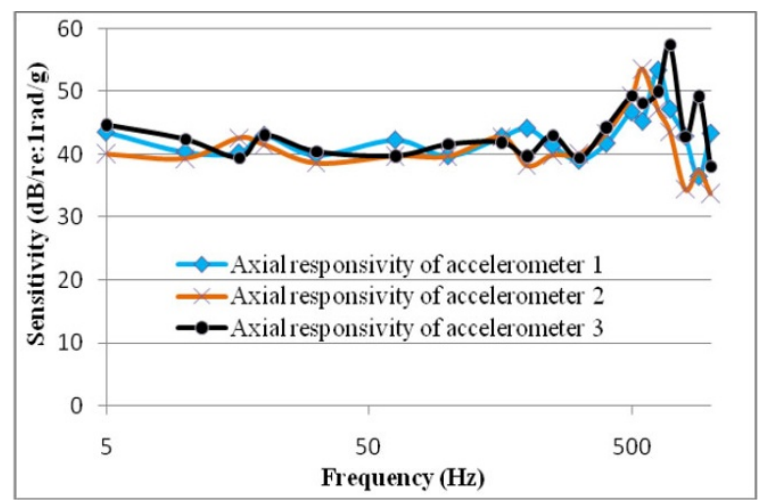

Fig. 8 Frequency sensitivity of the proposed accelerometer.

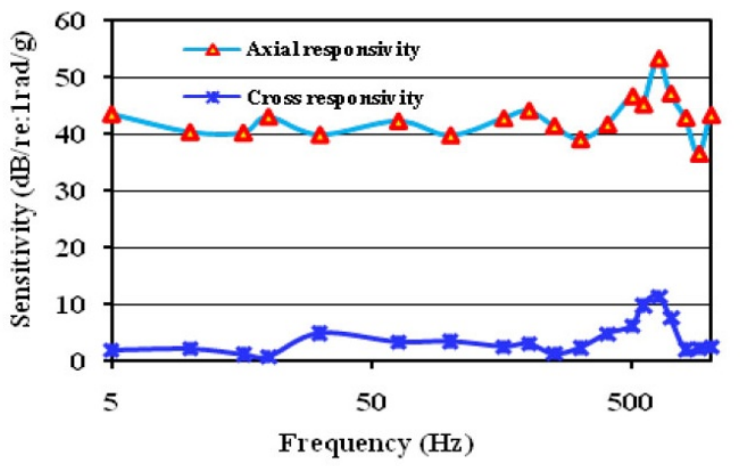

Fig. 9 Consistency of three accelerometers.

\section{Conclusions}

An all-metal double diaphragm-based optical fiber 3-component accelerometer with the low transverse sensitivity is proposed in this paper. The theoretical analysis is given based on the electro-mechanical theory. The sensitivity and resonance frequency of the sensor can be simultaneously enhanced by increasing the number of turns of the optical fiber without increasing the accelerometer size at the mass of a certain value. Experimental results show the good consistency of the proposed accelerometer. The proposed optical fiber seismometer is a good candidate for the seismic wave monitoring system in oil and gas exploration.

\section{Acknowledgement}

The authors thank the support of 863 Program of China (2013AA09A413 amd 2014AA093406), Beijing Nova Program (2010B055), and Key Instrument Developing Project of the Chinese Academy of Sciences (ZDYZ2012-1-08-03).

Open Access This article is distributed under the terms of the Creative Commons Attribution License which permits any use, distribution, and reproduction in any medium, provided the original author(s) and source are credited.

\section{References}

[1] P. R. Keul, E. Mastin, J. Blanco, M. Maguérez, T. Bostick, and S. Knudsen, "Using a fiber-optic seismic array for well monitoring," The Leading Edge, 2005, 24(1): 68-70.

[2] Y. Zhang, S. Li, J. Ning, Z. Yin, and H. Cui, "Seismic wave detection system based on fiber optic sensor," in Proc. SPIE, vol. 6296, pp. 62961J-1-2961J-8, 2006.

[3] N. Zeng, C. Shi, M. Zhang, L. Wang, Y. Liao, and S. Lai, "A 3-component fiber-optic accelerometer for well logging," Optics Communications, 2004, 234(1-6): 153-162.

[4] S. Knudsen, G. B. Havsgard, A. Berg, H. Nakstad, and T. Bostick, "Permanently installed high-resolution fiber optic 3C/4D seismic sensor systems for in-well imaging and monitoring applications," in Sixth Pacific Northwest Fiber Optic Sensor Workshop, pp. 51-55, 2003.

[5] J. M. De Freitas, "Recent developments in seismic seabed oil reservoir monitoring applications using fibre-optic sensing networks," Measurement Science and Technology, 2011, 22(5): 52001-52030.

[6] T. J. Hofler and S. L. Garrett, "Flexural disk fiber optic hydrophone," U.S. Patent 4959539, 1990.

[7] G. Chen, X. Zhang, G. Brambilla, and T. Newson, "Theoretical and experimental demonstrations of a microfiber-based flexural disc accelerometer," Optics Letters, 2011, 36(18): 3669-3671.

[8] G. A. Cranch and P. J. Nash, "High-responsivity 
fiber-optic flexural disk accelerometers," Journal of Lightwave Technology, 2000, 18(9): 1233-1243.

[9] S. Vohra, B. Danver, A. Tveten, and A. Dandridge, "Fiber optic interferometric accelerometers," in AIP Conference Proceedings, pp. 285-293, 1996.

[10] T. Vohra, B. Danver, A. Tveten, and A. Dandridge, "High performance fibre optic accelerometers," Electronics Letters, 1997, 33(2): 155-157.

[11] Q. Lin, L. Chen, S. Li, and X. Wu, "A highresolution fiber optic accelerometer based on intracavity phase-generated carrier (PGC) modulation," Measurement Science and Technology, 2011, 22(1): 15303-15308.

[12] Y. Wang, J. He, L. Feng, F. Li, and Y. Liu, “A DFB fiber laser sensor system using a NI-CompactRIO-based PGC demodulation scheme," in International Symposium on Photoelectronic Detection and Imaging 2009: Material and Device Technology for Sensors, vol. 7381, pp. 738110-1-73811O-6, 2009. 